\title{
Optimization model of a structural simulation design for a CICC
}

\author{
JIANG HuaWei ${ }^{*}$, WU SongTao ${ }^{2}$ \& CHENG JunSheng ${ }^{3}$ \\ ${ }^{1}$ College of Information Science and Engineering, Henan University of Technology, Zhengzhou 450001, China; \\ ${ }^{2}$ Institute of Plasma Physics, Chinese Academy of Sciences, Hefei 230031, China; \\ ${ }^{3}$ Institute of Electrical Engineering, Chinese Academy of Sciences, Beijing 100080, China
}

Received August 8, 2010; accepted January 10, 2011

\begin{abstract}
The engineering design of a Cable-In-Conduit Conductor (CICC) is complicated. A model for the optimal design of a CICC based on conductor stability, AC loss and strain is proposed. The model considers the critical current density as a function of applied strain. A mathematical programming method that minimizes the AC loss of the CICC is established to yield an optimal design for the CICC structure. The optimized structure and related performance agree well with the engineering design values used for the KSTAR project.
\end{abstract}

cable-in-conduit conductor, strain, numeric simulation design, stability, optimization model

Citation: Jiang H W, Wu S T, Cheng J S. Optimization model of a structural simulation design for a CICC. Chinese Sci Bull, 2011, 56: 2978-2983, doi: $10.1007 / \mathrm{s} 11434-011-4408-0$

The Cable-in-Conduit Conductor (CICC), which evolved from the Internally-Cooled Superconductor (ICS), has attracted attention to researchers since 1975 [1]. Initially, Chester made recommendations to improve the performance of the conductor, and later, Altov proposed several conductor structures [1-3]. Hoenig and others proposed the concept of a Cable-in-Conduit (CIC) and the model of the CICC based on experimental research [1-3]. With the advantages of supercritical helium cooling, high voltage insulation, low AC loss and multistage cabling, the CICC has been selected as the preferred conductor for use to Toroidal Fields (TFs), Poloidal Fields (PFs) in Experimental Advanced Superconducting Tokamak (EAST, in China), Korean Superconducting Tokamak Advanced Research (KSTAR) and International Thermal Nuclear Experimental Reactor (ITER).

The CICC is made of superconducting strands, stabilizer (copper), forced flow helium and jacket. The heat exchange between these components is very complex, and the influence on stability and AC loss are other major problems associated with CICCs. Accepting that the engineering design theory of CICCs is complex, there is a need to carry out

*Corresponding author (email: lhwcad@ sohu.com) further research through simulation, design and theoretical study of CICCs.

The simulation design and quenching of a CICC has been carried out. The Cable Interactive Designer (CID) is the main software tool used to simulate the cabling process [4], and is able to generate the geometry of cables. The 3D numerical simulation method proposed for the CICC, contains sub-cable wrapping and central cooling [5]. The numerical code Gandalf introduced quench simulation and analysis of the stability of a CICC cooled by forced flow helium [6]. Bottura presented basic guidelines for the effective design of a CICC based on parameters that included stability margin, AC response (losses and current distribution) and protection issues (hot-spot temperature and maximum quench pressure) [7]. In this study, the CID designs the CICC by simple mechanical iteration of the temperature margin and current conditions to obtain the structure of the conductor. The design is not based on energy margin. Gandalf was mainly concerned with the simulation and analysis of the stability and quenching of the CICC, but was not involved in the structural design of the CICC.

High Temperature Superconductors (HTS) have been applied to obtain a high magnetic field under the conditions of high current and a fast changing magnetic field [8], but 
this is still in the research stage and there are many scientific issues that need to be further explored. Recently, the $\mathrm{Nb}_{3} \mathrm{Sn}$ conductor has been developed based on niobium titanium (NbTi) technology. However, the strain caused by heat, mechanical and other factors is a key problem that is widely observed. It is a response to the spread and dissemination of heat energy in a CICC, and gives rise to the quench phenomenon. It is clear that strain can influence the operation of a CICC. There has been some progress in the theoretical investigation of these problems. The sensitivity of strain to critical current density is accurately described in [9], which also analyzes the deterioration of the critical current density and the strain effects on the $n$-value ( $n$-value is the changing rate of the synchronization back pinning when superconductor transforming the superconducting state into the normal state). Zhai et al. focused on the $\mathrm{Nb}_{3} \mathrm{Sn}$ CICC performance degradation problem of ITER, and the mixed-type magnetic field caused by the thermal and bending strains, proposing a high-performance Florida ElectroMechanical Cable Model (FEMCAM) by performing periodic load tests $[10,11]$. These tests can determine the deterioration situation for the $n$-value and critical current where the bending strains are less than $1 \%$. Muller et al. concluded that the brittleness of $\mathrm{Nb}_{3} \mathrm{Sn}$ results from bending and heat treatment [12]. The effects of strain on the critical current density, the critical field, critical temperature and $n$-values were elaborated on. While these studies obtained the deterioration of the $n$-value and the critical current for a CICC by experiment and simulation, they failed to apply these findings to the simulation design of the CICC structure through backward reasoning.

Jiang and $\mathrm{Wu}[13]$ carried out quantitative research on the strain effect on critical current density and other parameters, and built a numerical simulation design model of a CICC. However, in the process of simulation design based on layers (levels), the strand number of each layer is not adequately determined, which results in the strand number of a previous layer being much larger than the strand number of the subsequent layer. The proposed model cannot be used in practice because it cannot satisfy the requirements of mechanical structure stability (shown in section 3.3). The work of Jiang and $\mathrm{Wu}$ and other similar studies seemingly did not provide an adequate rational structure. This paper proposes a model for the optimal design of the CICC based on stability, AC loss and strain. The model considers the critical current density as a function of strain, and a mathematical programming method minimizing the AC loss is established to yield the optimal design of the CICC structure.

\section{Stability of the CICC}

\subsection{Model of CICC stability: Zero-dimension and one-dimension}

Accurate calculation of the stability margin of a short CICC sample is based on a number of factors. The length in the heating zone of the short CICC sample can be ignored assuming that the temperature of a strand, and that of the liquid helium, is uniform. Time is the only variable. The stability margin can be obtained in a short and a long pulse period. The equations of the zero-dimension model, which describes the local energy balance during recovery, can be acquired [14].

However, for a long CICC conductor with high transverse resistance, it is very difficult to re-distribute the current. The liquid helium temperature gradient in the CICC cross section leads to a dramatic change in the stability margin, so the uniformity assumption is no longer valid. Considering the effect on the stability of conduit transient thermal diffusion, the incompressible fluid equations can be obtained based on knowledge of the fluid (including mass, momentum and energy balance), and a one-dimensional model system can be established [14]. The model is used mainly to calculate the stability margin and predict the thermal hydraulic quenchback for multi-value stability. However, the numerous parameters that need to be analyzed, the large and slow calculations of the stability, quench, and other issues, all need to be resolved.

\subsection{Calculation of CICC Stability}

It is well-known that stability is a key parameter in the design process of a conductor for a superconducting magnet. According to [15], the stability margin of the CICC is limited by the available helium enthalpy between the sharing current temperature and the ambient helium temperature. The expression can be written as

$$
\Delta H=\frac{A_{\mathrm{he}}}{A_{\mathrm{sc}}} \int_{T_{\mathrm{op}}}^{T_{\mathrm{cs}}} C_{\mathrm{he}} \mathrm{d} T,
$$

where $A_{\text {he }}$ and $A_{\text {sc }}$ are the helium cross section and cable cross section. $T_{\mathrm{cs}}$ and $T_{\mathrm{op}}$ are the sharing current temperature and operating temperature and $C_{\mathrm{he}}$ is the specific heat of helium.

Based on the energy balance of the disturbance duration, a rough estimate of the energy margin can be given in the well-cooled, transition and ill-cooled regimes [15].

\section{Relationship between strain and critical current density}

The stability of the CICC not only depends on the magnetic field strength, temperature, current density distribution, angle between the strand and the magnetic field, but also on the strain on the strand (sub-cable). In the simulation design for the CICC, it is necessary to study the quantitative relationship between the $\mathrm{Nb}_{3} \mathrm{Sn}$ conductor strain and the critical current density. 


\subsection{Strain model}

The strain parameters mainly refer to effects caused by thermal and mechanical energy, which are key factors affecting the critical current density. Assuming that the mechanical strain of the CICC conduit will transfer to the strand, the effective strain on $\mathrm{Nb}_{3} \mathrm{Sn}$ can be written as the sum of three parts:

$$
\varepsilon=\varepsilon_{\mathrm{op}}+\varepsilon_{\mathrm{th}}+\varepsilon_{\mathrm{extra}}
$$

where $\varepsilon_{\mathrm{op}}$ is longitudinal strain in the conductor (also known as the operating strain), $\varepsilon_{\mathrm{th}}$ is thermal strain with zero current, and $\varepsilon_{\text {extra }}$ is the additional strain from other causes. Strictly, $\varepsilon_{\text {op }}$ and $\varepsilon_{\text {extra }}$ make up the real longitudinal strain. In the actual design, $\varepsilon_{\text {extra }}$ and $n$-value are the important parameters of the conductor structure, whose change is related to the properties of the strand. The thermal strain $\varepsilon_{\text {th }}$ can be estimated from the experimental data.

\subsection{Relationship between strain and critical current density}

In [16], several expressions of parameterization $\left(J_{\mathrm{c}}\left(B_{0}, T, \varepsilon\right)\right)$ were reviewed and compared. According to the literature $[16,17]$, combining the given magnetic field, the strain energy function with the formula for the CICC enthalpy estimates and the volume pinning force, the paper gives the following formula for the critical current density of $\mathrm{Nb}_{3} \mathrm{Sn}$ :

$$
J_{\mathrm{c}}\left(B_{0}, T, \varepsilon\right)=C(\varepsilon)\left(B_{\mathrm{C} 2}(T, \varepsilon)\right)^{-1 / 2}\left(1-t^{2}\right)^{2} b^{-1 / 2}(1-b)^{2},
$$

where $J_{\mathrm{c}}\left(B_{0}, T, \varepsilon\right)$ is the critical current density under the given conditions, $B_{0}$ is the operating background field, $T$ is the operating temperature, and $\varepsilon$ is the total longitudinal strain on the CICC.

$$
\begin{gathered}
C(\varepsilon)=C_{0}\left(1-a_{0}|\varepsilon|^{1.7}\right)^{1 / 2}, \\
B_{\mathrm{C} 2}(T, \varepsilon)=B_{\mathrm{C} 20}(\varepsilon)(1-t T)(1-t / 3), \\
b=B_{0} / B_{\mathrm{C} 2}(T, \varepsilon), \\
t=T / T_{\mathrm{C} 0}(\varepsilon), \\
T_{\mathrm{C} 0}(\varepsilon)=T_{\mathrm{C} 0 \mathrm{M}}\left(1-a_{0}|\varepsilon|^{1.7}\right)^{1 / 3}, \\
B_{\mathrm{C} 20}(\varepsilon)=B_{\mathrm{C} 20 \mathrm{M}}\left(1-a_{0}|\varepsilon|^{1.7}\right),
\end{gathered}
$$

where $\alpha_{0}=\left\{\begin{array}{cc}900 & \varepsilon<0 \\ 1255 & \varepsilon \geqslant 0\end{array}, C_{0}, T_{\mathrm{C} 0 \mathrm{M}}\right.$ and $B_{\mathrm{C} 20 \mathrm{M}}$ are gained from the experimental data under different conditions.

\section{Numerical simulation design of the CICC}

\subsection{Basic idea and assumptions}

Taking into account the given operating conditions, the stability margin, temperature margin and other parameters, considering the effect of strain on the critical current density, and the combination of the value of the Stekly parameter (Stekly parameter is the ratio of joule heat including other disturbance energy and heat transferred by liquid helium, that is, the ratio between the interference on conductor and cooling capacity of liquid helium per unit time. It is shown in the reference [18]), we derived a matrix equations. The equations contains the number of strands $\left(N_{\mathrm{sc}}\right)$, the superconducting strand diameter $\left(d_{\mathrm{sc}}\right)$, and the ratio between the copper and superconductor $\left(R_{\mathrm{cu}}\right)$. The equations also enabled us to configure the CICC.

In the design process, the following assumptions were made:

(1) The first sub-cable is twisted with three superconducting strands.

(2) The conductor operates in the transition regime in which the operating current is greater than the lower limiting current but does not exceed the upper limiting current. At the same time, the value of the Stekly parameter is less than one, namely, the heat energy is lower than the heat removed by the helium.

(3) When configuring the cable structure, to improve the structural stability of the CICC, any space will be filled with purity copper strands if the space defined by the diameter of an inscribed circle between the sub-cables is more than that of a sub-cable diameter.

\subsection{Simulation algorithm model of the CICC design}

In the transition regime $\left(I_{\text {lim,low }}<I_{\text {op }}<I_{\text {lim,up }}\right)$, based on the stability margin, temperature margin and space current density, the following equations can be derived [13]:

$$
\begin{gathered}
\frac{N_{\mathrm{sc}}^{2} d_{\mathrm{sc}}^{3} R_{\mathrm{cu}}}{R_{\mathrm{cu}}+1}=\frac{4 I_{\mathrm{op}}^{2} \rho_{\mathrm{cu}}}{\pi^{2} \mathrm{~K}_{\mathrm{p}} \alpha h\left(T_{\mathrm{c}}-T_{\mathrm{op}}\right)}=A, \\
R_{\mathrm{cu}}\left(R_{\mathrm{cu}}+1\right)=\frac{\rho_{\mathrm{cu}} \Delta E_{\mathrm{tr}} J_{\mathrm{c}}^{2}}{4 K_{\mathrm{p}} \mathrm{hC}_{\mathrm{he}}\left(T_{\mathrm{c}}-T_{\mathrm{op}}\right)^{2}} \frac{2 f_{\mathrm{v}}-1}{f_{\mathrm{v}}} d_{\mathrm{sc}}=B d_{\mathrm{sc}}, \\
\frac{R_{\mathrm{cu}}+1}{N_{\mathrm{sc}} d_{\mathrm{sc}}^{2}}=\frac{\pi J_{\mathrm{c}}}{4 I_{\mathrm{op}}}\left(1-\frac{\Delta T_{\mathrm{cs}}}{T_{\mathrm{c}}-T_{\mathrm{op}}}\right)=C,
\end{gathered}
$$

where $I_{\mathrm{op}}$ stands for the operating current, $\rho_{\mathrm{cu}}$ for the resistance of copper, $\pi$ for the circumference ratio, $K_{\mathrm{p}}$ for the coefficient of the wet perimeter, $\alpha$ for Stekly perimeter, $h$ for the heat transfer coefficient of helium, $T_{\mathrm{c}}$ for the critical temperature, $\Delta E_{\mathrm{tr}}$ for the stability margin in the transition regime, $f_{\mathrm{v}}$ for the void of the CICC, $\Delta T_{\mathrm{cs}}$ for the temperature margin, and $J_{\mathrm{c}}$ for the critical current density (the expression is shown in section 2.2).

\subsection{Numerical simulation design of CICC}

According to the above, eqs. (10)-(12), which include three 
unknown variables, may be solved. However, if eq. (12) is substituted into eq. (10), an equation similar to eq. (11) is acquired. This accounts for the fact that the optimization of the copper component of the CICC with the space current density is not an isolated factor, but is related to the stability and temperature margins. Consequently two equations with three unknown variables cannot be uniquely solved. This paper proposes a method in which $R_{\mathrm{cu}}$ and $d_{\mathrm{sc}}$ can be expressed as a function of $N_{\mathrm{sc}}$, the number of strands $N_{\mathrm{sc}}$ can be found gradually, based on the structure of the cable. Then, $R_{\mathrm{cu}}$ and $d_{\mathrm{sc}}$ can be solved according to specific requirements.

The expression of $R_{\mathrm{cu}}$ can be written as follows using eq. (12):

$$
R_{\mathrm{cu}}=C N_{\mathrm{sc}} d_{\mathrm{sc}}^{2}-1 .
$$

If eq. (13) is substituted into eq.(10), we obtain the following:

$$
d_{\mathrm{sc}}^{3}-\frac{1}{N_{\mathrm{sc}} C} d_{\mathrm{sc}}-\frac{A}{N_{\mathrm{sc}}}=0 .
$$

Based on a different configuration for the CICC, the value of $N_{\text {sc }}$ can be obtained, as well as the values of $d_{\text {sc }}$ and $R_{\mathrm{cu}}$.

Given the operating conditions: $B_{\max }=7.2 \mathrm{~T}, T_{\mathrm{op}}=4.5 \mathrm{~K}$, $I_{\mathrm{op}}=35200 \mathrm{~A}, \Delta T_{\mathrm{cs}}=1 \mathrm{~K}, \mathrm{~d} B / \mathrm{d} t=11 \mathrm{~T} / \mathrm{s}, \Delta E_{\mathrm{tr}}=400 \mathrm{~mJ} / \mathrm{cm}^{3}$, we have, after the simulation design of the CICC, the total number of strands that meet the conditions is 486 , which includes two cases. The structure of CICC with four cabling is shown in Table 1, where there are six cabling methods. The structure of the CICC with five cabling is shown in Table 2, where there are four cabling methods.

We can see from Tables 1 and 2 that the number of strands in each layer is not uniquely determined. They will be appear as structures of $3 \mathrm{SC} \times 3 \times 6 \times 9,3 \mathrm{SC} \times 6 \times 9 \times 3$ and other, so the structures of the simulation design of the CICC are not unique. For example, when it appears as $3 \mathrm{SC} \times 6 \times$

Table 1 the structure of CICC with four cabling

\begin{tabular}{ccc}
\hline Second sub-cable & Third sub-cable & Fourth sub-cable \\
\hline 3 & 6 & 9 \\
3 & 9 & 6 \\
6 & 3 & 9 \\
6 & 9 & 3 \\
9 & 3 & 6 \\
9 & 6 & 3 \\
\hline
\end{tabular}

Table 2 The structure of CICC with five cabling

\begin{tabular}{cccc}
\hline Second sub-cable & Third sub-cable & Fourth sub-cable & Fifth sub-cable \\
\hline 3 & 3 & 3 & 6 \\
3 & 3 & 6 & 3 \\
3 & 6 & 3 & 3 \\
6 & 3 & 3 & 3 \\
\hline
\end{tabular}

$9 \times 3$, we note that its third level number (9) is more than the fourth (3), and it cannot meet the requirements of mechanical structure stability. Therefore, we need to optimize the design to provide an acceptable CICC structure.

\section{Optimal design of the CICC}

\subsection{Structure for the optimization design of the CICC}

The principle and method of optimization is given. Firstly, the number of strands in each layer is acceptable if it can meet the requirements of mechanical structure stability. Then, we need to determine the AC losses of the CICC by calculating the coupling loss time constant, to find the minimum AC loss (including coupling and hysteresis loss) via iterative calculations, and thus, to acquire the optimized conductor structure.

To optimize the number of strands in each layer and the AC losses, according to the general optimization method, it need only meet the following mathematical programming conditions:

$$
\left\{\begin{array}{l}
\min \left(E_{\mathrm{c}}\right)=\min (W+P) \\
\text { s.t. } \quad n_{i} \leqslant n_{j} \quad(i<j)
\end{array},\right.
$$

where $n_{i}$ is the $i$ th sub-cable structure, which is also the number of sub-cables (strands). Therefore, we can determine that structures $3 \mathrm{SC} \times 3 \times 6 \times 9$ and $3 \mathrm{SC} \times 3 \times 3 \times 3 \times 6$ in Tables 2 and 3 can meet the required conditions.

The selected structure of the CICC is then optimized by calculating the coupling loss. Considering the coil excitation, plasma discharge or burst, and the extreme conditions, most superconducting wires in the CICC strands are saturated. Using the saturation coefficient, the coupling loss can be described as follows [19]:

$$
P= \begin{cases}\frac{2 \theta}{u_{0}} \dot{B}_{\mathrm{T}}^{2}(1-\beta) & \beta<0.31, \\ \frac{4}{3 \pi} \frac{B_{\mathrm{P}}}{u_{0}} \dot{B}_{\mathrm{T}} & \beta \geqslant 0.31,\end{cases}
$$

where $B_{\mathrm{T}}$ is the transverse field, and the saturation coefficient $\beta$ is the rate between the changing of the external magnetic field and the penetration field $B_{\mathrm{P}}, u_{0}$ is the magnetic permeability of vacuum.

It is clear that the coupling loss time constant is a key parameter. The coupling loss time constant $\theta$ can be calculated as follows:

$$
\theta=\sum_{n=1}^{\mathrm{N}} \theta_{n}
$$

where $\theta_{n}$ corresponds to the coupling loss time constant increase at the $n$th level, and its expression is given by 


$$
\theta_{n}=\frac{\mu_{0}}{2 \rho_{n}}\left(\frac{P_{n}^{*}}{2 \pi}\right)^{2} \frac{1}{1-f_{v n-1}}
$$

where $P_{n}^{*}, \rho_{n}$, and $f_{v n}$ are the effective cable length, the effective resistivity and the void of the $n$th level.

The effective cable length and the effective resistivity can be written as follows:

$$
\begin{gathered}
P_{n}^{*}=P_{n}-\frac{r_{n-1}}{R_{n-1}} P_{n-1}, \\
\rho_{n}=\frac{\rho_{\mathrm{b}} e_{\mathrm{b}}}{\varepsilon_{n} R_{n-1}},
\end{gathered}
$$

where $P_{n}, R_{n}, r_{n}$, and $\varepsilon_{n}$ are the corresponding cable length, the outer radius, the radius and the contact surface ratio of the $n$th level, $\rho_{\mathrm{b}} e_{\mathrm{b}}$ is the characterization of the contact resistance layer.

The coupling loss time constant of the two CICC can be obtained by simulation calculations (shown in Table 3). Taking the smaller value of the AC loss, the structure of $3 \mathrm{SC} \times 3 \times 3 \times 3 \times 6$ will be the more acceptable one.

The simulation optimization of the CICC structure is illustrated in Figure 1. From the figure we can see the configuration of the CICC. The first sub-cable contains three superconducting strands. The second sub-cable is made up of the three first sub-cables. In the third sub-cable there are three second sub-cables. The fourth sub-cable includes three third sub-cables. The six fourth sub-cables form the overall cable. Finally, the cable was embedded in a stainless steel conduit and configured into the squared shape.

Table 3 Calculation of the conductor structure

\begin{tabular}{ccc}
\hline Parameter & $3 \mathrm{SC} \times 3 \times 3 \times 3 \times 6$ & $3 \mathrm{SC} \times 3 \times 6 \times 9$ \\
\hline$\theta_{0}(\mathrm{~ms})$ & 0.8677 & 0.8677 \\
$\theta_{1}(\mathrm{~ms})$ & 1.3894 & 1.3894 \\
$\theta_{2}(\mathrm{~ms})$ & 3.6906 & 3.6906 \\
$\theta_{3}(\mathrm{~ms})$ & 8.1790 & 13.209 \\
$\theta_{4}(\mathrm{~ms})$ & 12.389 & 27.735 \\
$\theta_{5}(\mathrm{~ms})$ & 18.095 & $/$ \\
$\theta_{\mathrm{t}}(\mathrm{ms})$ & 44.611 & 46.892 \\
$E_{\mathrm{c}}\left(\mathrm{mJ} / \mathrm{cm}^{3}\right)$ & 203.24 & 217.37 \\
\hline
\end{tabular}

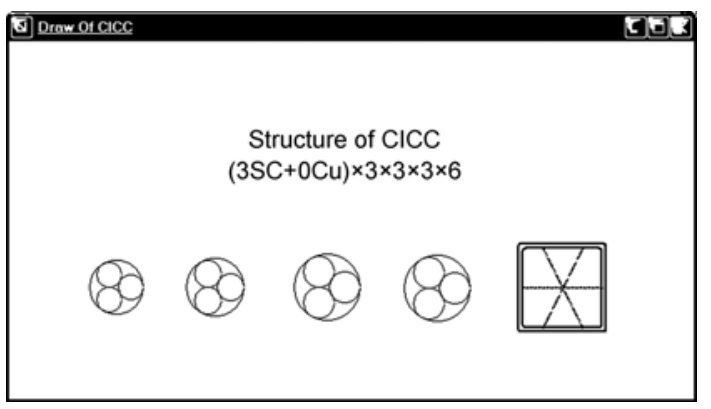

Figure 1 The result of the optimization simulation.

\subsection{Results and discussion}

To investigate the simulation design of the CICC, an experiment was carried out in the IPPCAS. We emphasized the contrast between the simulation design results and the engineering design of KSTAR. Under the given conditions, the data obtained from the simulation design of the CICC were compared with those of KSTAR as shown in Tables 4 and 5.

Table 4 shows that the simulation structure agrees well with the engineering design, which is the optimized design based on the stability, the effect of strain and the AC loss.

From Table 5 we can see that the AC losses of the optimized CICC are less than for the engineering design value, which indicates that the disturbance in the optimized CICC was fewer than in the engineering design. The energy margin for the optimized CICC was more than for the engineering design, so the optimized conductor is more stable. While the AC losses of the optimized CICC were fewer than for the engineering and simulation designs, it was shown that the optimized CICC, under the given conditions, can operate well.

It was also found that there were a few differences between the results of the numerical simulation and those of the engineering design as seen in Table 5. For instance, the difference in the copper cross section is controlled by different twisting coefficients. Moreover, there exist different critical currents between the simulation design and engineering design, which is caused by the method of simulation. The difference in the stability margin was a result of $A_{\mathrm{cu}}$. Some improvement is possible, such as by appropriately wetting the perimeter coefficient to make up for the simulation errors.

Table 4 Structure of the simulation compared with that of the KSTAR

\begin{tabular}{ccc}
\hline Configuration & $\begin{array}{c}\text { Value of engineering } \\
\text { design }\end{array}$ & $\begin{array}{c}\text { Result of numerical } \\
\text { simulation }\end{array}$ \\
\hline First sub-cable & $3 \mathrm{SC}$ & $3 \mathrm{SC}$ \\
Second sub-cable & $3 \mathrm{SC} \times 3$ & $3 \mathrm{SC} \times 3$ \\
Third sub-cable & $3 \mathrm{SC} \times 3 \times 3$ & $3 \mathrm{SC} \times 3 \times 3$ \\
Fourth sub-cable & $3 \mathrm{SC} \times 3 \times 3 \times 3$ & $3 \mathrm{SC} \times 3 \times 3 \times 3$ \\
CICC & $3 \mathrm{SC} \times 3 \times 3 \times 3 \times 6$ & $3 \mathrm{SC} \times 3 \times 3 \times 3 \times 6$ \\
\hline
\end{tabular}

Table 5 Parameters of the simulation compared with those of the KSTAR

\begin{tabular}{lcc}
\hline Parameter & $\begin{array}{c}\text { Value of engineering } \\
\text { design }\end{array}$ & $\begin{array}{c}\text { Result of numerical } \\
\text { simulation }\end{array}$ \\
\hline$A_{\mathrm{cu}}\left(\mathrm{mm}^{2}\right)$ & 170.3 & 167.6 \\
$A_{\mathrm{sc}}\left(\mathrm{mm}^{2}\right)$ & 61.9 & 60.2 \\
$T_{\mathrm{c}}(\mathrm{K})$ & 12 & 12.57 \\
$T_{\mathrm{cs}}(\mathrm{K})$ & $<10.2$ & 6.58 \\
$I_{\mathrm{c}}(\mathrm{A})$ & 80000 & 84488 \\
$d_{\mathrm{sc}}(\mathrm{mm})$ & 0.78 & 0.779 \\
$R_{\mathrm{cu}}$ & 1.5 & 1.51 \\
$\Delta E_{\mathrm{tr}}\left(\mathrm{mJ} / \mathrm{cm}^{3}\right)$ & 400 & 473.6 \\
$E_{\mathrm{c}}\left(\mathrm{mJ} / \mathrm{cm}^{3}\right)$ & $\leqslant 250$ & 203.24 \\
\hline
\end{tabular}




\section{Conclusions}

This paper puts forward the basic principle of numerical simulation design. Using the experience of the engineering design, a quantitative effect model of the strain on the critical current density was built. It was assumed that the first level sub-cable had three superconducting strands and the conductor operated in the transition regime.

According to the mechanism determining the number of sub-cables (strands), a mathematical programming method that contained the AC loss and other variables was established, and a reasonable structure for a conductor was provided using the optimization method.

The results of the numerical simulation were compared with those of the engineering design, and the errors is analyzed. The simulation results are encouraging and the numerical simulation design can clearly help in the design of the CICC.

The authors would like to express their appreciation to Profs. Qiu Liang Wang and $Y u$ Wu for their help. We also gratefully thank the EAST team for their support.

1 Dresener L. Twenty years of cable-in-conduit conductors: 1975-1995. J Fus Energ, 1995, 14: 3-12

2 Seeber B. Hand Book of Applied Superconductivity. London: Institute of Physics Publication, 1998

3 Renard B, Martinez A, Duchateau J L, et al. Transverse heat transfer coefficients on a full size dual channel CICC ITER conductor. Cryogenics, 2006, 46: 530-540

4 Bottura L, Breschi M, Rosso C. Analysis of electrical coupling parameters in superconducting cables. Cryogenics, 2003, 43: 233-239
5 Qin J G, Wu Y. A 3D numerical model study for superconducting cable pattern. Fus Eng Des, 2010, 85: 109-114

6 Marinucci C, Bottura L, Vecsey G, et al. The quell experiment as a validation tool for the numerical code gandalf. Cryogenics, 1998, 38: 467-477

7 Bottura L. Stability, protection and ac loss of cable-in-conduit conductors-a designer's approach. Fus Eng Des, 1993, 20: 351-362

8 Ma T C, Wan Y, Jiang S. Research progress in synthesis of iron-based superconducting materials. Chinese Sci Bull, 2009, 54: 557-567

9 Zhang P X, Liang M, Tang X D, et al. Strain influence on $J_{\mathrm{c}}$ behavior of $\mathrm{Nb}_{3} \mathrm{Sn}$ multifilamentary strands fabricated by internal tin process for ITER. Physic C, 2008, 46: 1843-1846

10 Zhai Y H, Mark D B. Florida electro-mechanical cable model of $\mathrm{Nb}_{3} \mathrm{Sn}$ CICCs for high-field magnet design. Supercond Sci Technol, 2008, 21: 1-15

11 Zhai Y H. Electro-mechanical modeling of $\mathrm{Nb}_{3} \mathrm{Sn}$ CICC performance degradation due to strand bending and inter-filament current transfer. Cryogenics, 2010, 50: 149-157

12 Muller H, Schneider T H. Heat treatment of $\mathrm{Nb}_{3} \mathrm{Sn}$ conductors. Cryogenics, 2008, 48: 323-330

13 Jiang $\mathrm{H} \mathrm{W}$, Wu S T. Research of simulation design model for CICC based on strain. Acta Electron Sin, 2010, 38: 1334-1388

14 Bottura L, Luongo C. Superconductors, stability in forced flow. In: John G W, ed. Wiley Encyclopedia of Electrical and Electronic Engineering. Milton: John Wiley \& Sons, 1999. 126-158

15 Jiang H W, Wu S T. Research of simulation design for CICC based on energy margin and temperature margin. IEEE Trans Appl Supercond, 2010, 20: 1436-1439

16 Bottura L, Bordini B. $J_{\mathrm{c}}(B, T, \varepsilon)$ parameterization for the ITER $\mathrm{Nb}_{3} \mathrm{Sn}$ production. IEEE Trans Appl Supercond, 2009, 19: 1521-1524

17 Summers L T, Guinan M W, Miller J R, et al. A model for the prediction of $\mathrm{Nb}_{3} \mathrm{Sn}$ critical current as a function of field, temperature, strain and radiation damage. IEEE Trans Magn, 1991, 27: 20412044

18 Wilson M N. Superconducting Magnets. Oxford: Oxford University Press, 1983

19 Fang J, Weng P D, Chen Z M, et al. The ac losses measurement and analysis of superconducting NbTi CICC for HT-7U superconducting Tokamak. Plasm Sci Technol, 2003, 14: 76-82

Open Access This article is distributed under the terms of the Creative Commons Attribution License which permits any use, distribution, and reproduction in any medium, provided the original author(s) and source are credited. 